\title{
Randomized Controlled Trial on the Effectiveness of Counseling Messages for Avoiding Unprotected Sexual Intercourse During Sexually Transmitted Infection and Reproductive Tract Infection Treatment Among Female Sexually Transmitted Infection Clinic Patients
}

\author{
Clive Anderson, MD*, Maria F. Gallo, $\mathrm{PhD}^{\dagger}$, Tina Hylton-Kong, MBBS ${ }^{\star}$, Markus J. Steiner, \\ PhD $\ddagger$, Marcia M. Hobbs, PhD§, Maurizio Macaluso, MD ${ }^{\dagger}$, , J. Peter Figueroa, MBBS $\|^{\|}$, Denise \\ J. Jamieson, $\mathrm{MD}^{\dagger}$, Jennifer Legardy-Williams, $\mathrm{MPH}^{\dagger}$, Jeffrey Wiener, $\mathrm{PhD}^{\dagger}$, and Lee Warner, \\ $\mathrm{PhD}^{\dagger}$ \\ ${ }^{*}$ Comprehensive Health Centre/Epidemiology Research and Training Unit, Jamaica Ministry of \\ Health, Kingston, Jamaica \\ tDivision of Reproductive Health, Centers for Disease Control and Prevention, Atlanta, GA \\ ¥Clinical Sciences Division, Durham, NC \\ $\S$ Department of Medicine, University of North Carolina at Chapel Hill, Chapel Hill, NC \\ IDivision of Biostatistics and Epidemiology, Cincinnati Children's Hospital Medical Center, MLC, \\ Cincinnati, $\mathrm{OH}$ \\ "Department of Community Health and Psychiatry, University of West Indies, Mona, Kingston, \\ Jamaica
}

\begin{abstract}
Background-The effectiveness of counseling messages to avoid unprotected sex during shortterm treatment for curable sexually transmitted infections is unknown.

Methods-We randomized 300 female STI clinic patients 18 years or older with cervicitis and/or vaginal discharge in Kingston, Jamaica, in 2010 to 2011, to 1 of 2 counseling messages for their course of syndromic treatment: abstinence only or abstinence backed up by condom use. At a follow-up visit 6 days afterward, we collected vaginal swabs to test for prostate-specific antigen (PSA), a biological marker of recent semen exposure, and administered a questionnaire assessing sexual behavior.
\end{abstract}

Results-No differences were found in the proportions of women testing positive for PSA at follow-up in the abstinence-plus-condom group (11.9\%) and abstinence-only group (8.4\%) (risk difference, $3.5 ; 95 \%$ confidence interval, -3.5 to 10.5 ). There also was no significant difference in reporting of unprotected sex between groups. Reporting a history of condom use before enrollment significantly modified the effect of counseling arm on PSA positivity $(P=0.03)$. Among those reporting recent condom use, $10.3 \%$ in the abstinence-only arm and $4.8 \%$ in the abstinence-plus-condom arm tested positive for PSA. Conversely, among those not reporting

Copyright () 2013 American Sexually Transmitted Diseases Association All rights reserved

Correspondence: Maria F. Gallo, PhD, Division of Reproductive Health, 4770 Buford Highway, Mail Stop K-34, Atlanta, GA 30341-3724 mgallo@cdc.gov.

The authors have no conflicts of interests. 
recent condom use, $6.5 \%$ in the abstinence-only arm and $17.3 \%$ in the abstinence-plus-condom arm had PSA detected.

Conclusions-We found no evidence to support the superiority of either counseling message. Post hoc analyses suggest that women with recent condom experience may benefit significantly more from abstinence-plus-condom messages, whereas women without such experience may benefit significantly more from abstinence-only messages. Providers should weigh individual condom use history when determining the most appropriate counseling message.

The World Health Organization estimates 340 million new cases of sexually transmitted infections (STIs) occur annually worldwide, with the rate of new infections in Latin America and the Caribbean second only to that of sub-Saharan Africa. ${ }^{1}$ Because STIs increase risk for adverse health outcomes such as HIV,, 3 preventing patients with newly diagnosed, curable STIs from infecting their partners is crucial. The main strategy for preventing secondary infections involves counseling index patients either to abstain from sex while they remain infectious or to use condoms consistently and correctly to protect against the transmission of STIs spread primarily through skin-to-skin contact or, to a lesser degree, those spread by contact with mucosal surfaces. ${ }^{4}$

The effectiveness of abstinence-only interventions, in general, has not been demonstrated. ${ }^{5}$ although abstinence-based interventions combined with safer-sex strategies such as condom use may reduce self-reported behaviors, data on their effectiveness against biological outcomes is limited. ${ }^{6}$ Furthermore, evidence from the few trials conducted, which involve long-term behavior changes, might not be applicable in the context of preventing transmission from patients with curable infections during their short-term course of treatment. Guidelines from the Centers for Disease Control and Prevention and other bodies recommend complete abstinence but do not recommend or mention the use of condoms in this situation. ${ }^{4,7}$ Conversely, the Jamaican Ministry of Health recommends both abstinence and condom use interventions in their written guidance, which has resulted in patients receiving either of 2 counseling messages for the treatment period: abstinence or abstinence backed up by condom use in case sex was to occur. ${ }^{8}$ Given their unknown relative effectiveness, we conducted a pilot randomized controlled trial using a biological marker of recent semen exposure detected in vaginal fluid as a proxy of recent unprotected vaginal intercourse in addition to self-reported sexual behavior to compare these 2 counseling messages.

\section{MATERIALS AND METHODS}

\section{Participants and Procedures}

The Assessing Counseling Message Effectiveness Study randomized 300 women attending a public STI clinic in Kingston, Jamaica, from August 2010 to March 2011, to receive 1 of 2 counseling messages: (1) a single message promoting short-term abstinence ("abstinence only") or (2) a hierarchical message promoting abstinence backed up by the promotion and provision of condoms ("abstinence plus condom"). Eligible women were at least 18 years of age, prescribed syndromic treatment for cervicitis and/or vaginal discharge according to standard clinical care (and hence were eligible to receive a counseling message for abstaining from coitus), and had no antibiotic use in the past 14 days. Pregnant or HIVpositive women were ineligible because these populations could have differed in unknown ways from other women with respect to likelihood of adherence to the counseling messages.

We prepared sequentially numbered and sealed, opaque envelopes containing the randomized assignment using permuted block randomization with the order generated by a pseudorandom number generator using a fixed, preselected parameter. Although masking 
participants and clinic study staff to arm assignment was not feasible, staff analyzing the data and laboratory staff remained masked until the analyses were completed.

We assessed prospective participants who gave written consent for screening for pregnancy and HIV status and performed a pelvic examination. Patients found to be syndromically positive for cervicitis and/or vaginal discharge received the recommended treatment regimen specified by the Jamaican Ministry of Health. ${ }^{8}$ Standard clinical practice was followed for referring their partners for care. A study clinician collected vaginal swabs for immediate testing for STIs and for later testing for prostate-specific antigen (PSA), which is a biomarker of semen detectable for up to 48 hours postexposure. ${ }^{9}$ Women eligible after screening could give written consent for study enrollment. The clinician read the assigned counseling message aloud to participants using scripts standardized for the trial (Panel). Staff verbally administered a quantitative questionnaire to collect information on demographics, sexual activity, and condom use. We decided a priori not to use audio computer-assisted self-interviewing to collect the quantitative data because this method has not been definitively proven to improve the validity of self-reports of sexual behavior. ${ }^{10}$

A single follow-up visit was scheduled 6 days after enrollment to coincide with the time most participants would still be on syndromic treatment and thus subject to adhere to their assigned counseling message. At the follow-up visit, staff again collected vaginal swabs for STI and later PSA testing and administered a questionnaire. At the end of the visit, we asked the participants for written consent to retroactively test their already collected vaginal swabs from both the enrollment and follow-up visits for the presence of PSA. We used this sequence of procedures to minimize bias from participants modifying their sexual behavior or their reporting of sexual behavior, (independent of the counseling message provided) as a result of having prior knowledge of the biomarker testing. We subsequently destroyed swabs from participants who did not provide consent for retroactive PSA testing. Ethical review boards at the Jamaican Ministry of Health and the Centers for Disease Control and Prevention approved the research.

\section{Laboratory Testing}

We tested vaginal swabs collected from the 2 study visits on-site with ABAcard $\mathrm{p} 30$ (Abacus Diagnostics, West Hills, CA; 4.50 per test), a rapid, semiquantitative test for PSA, following published procedures. ${ }^{11}$ In brief, vaginal swab eluate was added to the sample well of the cassette and allowed to incubate for 10 minutes at room temperature. Cassettes with a visible line in the control area but not the test area were interpreted as negative for PSA, whereas those with visible test and control lines were graded as low or high positive for PSA based on the intensity of the pink test line compared with PSA standards. High positives corresponded to standard solutions containing $23 \mathrm{ng}$ PSA/mL or greater; low positives corresponded to standard solutions containing 4 to less than $23 \mathrm{ng}$ PSA $/ \mathrm{mL}$. We performed quality assurance by testing a second swab from the first 82 participants using a quantitative test (Abbott Diagnostics, Abbott Park, IL) at the University of North Carolina at Chapel Hill. Using the quantitative test as the reference standard, we found $100 \%$ sensitivity and $98.8 \%$ specificity for the ABAcard results when their outcomes were classified as low or high positive versus negative and 100\% sensitivity and $100 \%$ specificity when their outcomes were classified as high positive versus low or negative.

We diagnosed STIs on-site using the APTIMA Combo2 Assay (Gen-Probe, Inc.; San Diego, CA) for detection of Neisseria gonorrhoeae and Chlamydia trachomatis nucleic acids according to the manufacturer's instructions. Trichomonas vaginalis nucleic acids were detected using the APTIMA TV Analyte Specific Reagents (Gen-Probe, Inc.) as previously described. ${ }^{12}$ 


\section{Statistical Analysis}

To compare the relative effectiveness of the 2 counseling messages, we calculated the risk difference (RD), with a 95\% confidence interval (CI) of testing positive for PSA at followup. We used an intent-to-treat analysis of participants with non-missing PSA data from the follow-up visit; sensitivity analyses included restricting the analysis to the per-protocol population of participants who attended their follow-up visit on the scheduled date or 1 day before or after. We also evaluated outcomes based on (1) reclassifying the PSA test result to negative or low positive PSA versus high positive and (2) using self-reported lack of unprotected vaginal sex instead of the biological outcome.

Based on limited evidence from studies measuring the proportions of women testing positive for PSA, ${ }^{10,13-16} 2$ of which were conducted among non-sex worker populations, ${ }^{10,16}$ we estimated that $20 \%$ of participants would test positive for PSA at the follow-up visit. Using a 2-tailed test with a value of 0.025 and $80 \%$ power, a sample size of 300 would permit the rejection of the null hypothesis of no difference between arms if the incidence of PSA in one arm were $20 \%$ and the incidence in the more effective arm were $8 \%$ (i.e., RD of 0.12 ). We followed CONSORT guidelines for the reporting of the results. ${ }^{17}$

\section{RESULTS}

\section{Study Population}

Of 495 women attending the STI clinic who were invited to screen for the study, 116 were not interested in learning about the study, 23 women declined to consent, and 56 did not meet the eligibility criteria (Fig. 1). Thus, we enrolled and randomized 300 women in the study in a balanced ratio (150 in each arm) to receive one of the counseling messages. Seven women failed to return for follow-up, and an additional 7 declined consent for PSA testing. Consequently, the intent-to-treat population consisted of 286 women (95.3\% of those randomized), equally balanced by study arm (143 in each arm).

Altogether $29 \%$ of the participants were single, $36 \%$ had a visiting partner (understood as a conjugal partner who does not live with her), ${ }^{18}$ and $34 \%$ were married or cohabiting (Table 1). Participants had a median of 11 years of education, 2 prior births, and 1 sexual partner in the past 3 months. Women in the abstinence-only arm were slightly younger than those in the abstinence-plus-condom arm (median of 27 and 29 years, respectively). Women in the abstinence-only arm were more likely to report having used a condom in the past 3 months compared with women in the abstinence-plus-condom arm (56\% vs. $43 \%$, respectively). Similarly, women in the abstinence-only arm were more likely to report currently using condoms for contraception compared with those in the abstinence-plus-condom arm (31\% vs. $19 \%$, respectively).

\section{Effectiveness of Counseling Messages}

Approximately $10 \%$ of participants overall had biologic evidence of recent unprotected intercourse detected at the follow-up visit. The proportion of women with PSA detected was slightly higher in the abstinence-plus-condom group (11.9\%) compared with the abstinenceonly group (8.4\%), although the difference was not statistically significant (RD, 3.5; 95\% CI, 3.5-10.5) (Table 2). Adjusting for potential confounders included in Table 1 had little effect on the primary effect estimate (data not reported). Repeating the analyses using the per-protocol population (i.e., restricting the analysis to those who returned 5-7 days after enrollment) had only a minimal effect on estimates (data not shown). No significant differences were found between groups in analyses of any other of the measured secondary outcomes (Table 2), including those based on self-reported sexual behavior. For example, there was no significant difference in reporting of unprotected sex since the enrollment visit 
between the abstinence-plus-condom group (17.9\%) and the abstinence-only group (11.7\%) (RD, 6.2; 95\% CI, -2.1 to 14.5$)$. Overall, $19.6 \%(\mathrm{n}=56)$ of women $(23.1 \%$, abstinence plus condom; $16.1 \%$ abstinence only) at the follow-up visit reported having unprotected vaginal sex since enrollment or tested positive for PSA.

Reporting a history of condom use during sex in the 3 months before enrollment significantly modified the effect of the counseling arm on whether PSA was detected at the follow-up visit $(P=0.03)$. Among 140 women who reported using a condom during sex in the past 3 months, $4.8 \%$ in the abstinence-plus-condom arm and $10.3 \%$ in the abstinenceonly arm tested positive for PSA at follow-up (RD, $-5.4 \%$; 95\% CI, $-14.0 \%$ to $3.2 \%$ ). In contrast, among 143 women who reported no condom use during the past 3 months, $17.3 \%$ in the abstinence-plus-condom arm and 6.5\% in the abstinence-only arm had PSA detected at follow-up (RD, $10.8 ; 95 \% \mathrm{CI}, 0.6 \%-21.1 \%)$.

\section{DISCUSSION}

Overall, we found no evidence in the randomized comparisons to support the superiority of either of the 2 counseling messages for avoiding unprotected sex during the treatment period for selected curable STIs or reproductive tract infections in this pilot randomized controlled trial. The proportion of female STI clinic patients who were counseled on abstinence or abstinence backed up by condom use did not significantly differ in their risk of having a biomarker of semen exposure detected approximately 1 week after being prescribed treatment or in self-reported sexual behavior. The present study differed from prior studies of behavioral interventions among patients at STI clinics ${ }^{19,20}$ in 2 regards: the use of an objective biomarker of semen exposure and the focus on sexual behavior during the short period of STI treatment.

Our findings from a post hoc analysis suggest that counseling message effectiveness was modified by participants' recent experience using condoms. Women with experience with condoms may benefit significantly more from the hierarchical message that included condoms, whereas women without such experience may benefit significantly more from the abstinence-only message. A similar effect regarding male condom use at baseline was found in a randomized crossover trial comparing the female condom with the male condom among women attending a family planning clinic in Brazil. ${ }^{21}$ Although the 2 condom types in that study had similar rates of moderate or high concentrations of PSA detected in postcoital specimens, women who reported experience at baseline with using male condoms appeared to have an increased risk of PSA when they used the female condom.

These findings suggest that providers should weigh the patient's condom use history when determining the best counseling message and prevention approach for an individual patient. Client-centered counseling has previously been shown to be associated with reduced rates of STIs and increased rates of self-reported condom use. ${ }^{22}$ Future research could use semen biomarkers to evaluate the effectiveness of client-centered counseling, especially among patients with a syndromic or laboratory-confirmed STI diagnosis who currently already receive routine counseling.

The lack of a significant difference observed between counseling messages could be attributable to several factors. For example, the brief counseling messages administered could have been insufficient to differentially influence sexual behavior among patients recently diagnosed as having infection above and beyond existing preventive approaches. The Jamaican Ministry of Health has a comprehensive HIV program, which already includes educational campaigns on condom use and social marketing of condoms. ${ }^{23}$ Thus, any differences between the 2 counseling messages could have been diluted to the extent that 
participants in the abstinence-only arm were exposed to condom messages or condom products outside the study context.

Given that the $10 \%$ observed incidence of PSA positivity was markedly lower than anticipated, the pilot trial likely was underpowered to detect statistically significant differences between intervention arms. Although we were able to ascertain whether a large difference existed in the effectiveness of the 2 messages, further research would be needed to rule out a smaller difference. Furthermore, despite providing comparative evidence of relative effectiveness, the trial results are non-informative as to the magnitude of effectiveness of the counseling messages for avoiding unprotected vaginal sex during the treatment period. The proportion of women with a positive PSA biomarker detected at follow-up is an underestimation of the proportion who actually engaged in unprotected sex during the observation period, as an estimated $71 \%$ of exposed women will test negative by 24 hours, and by 48 hours, almost none will have identifiable PSA. ${ }^{9}$ Thus, the observed PSA rate of $10 \%$ should be interpreted as the lower bound of the frequency of exposure during the treatment interval, which suggests that an intervention more intensive than the brief counseling messages studied here may be needed to modify women's sexual behavior. We noted, however, that similar study results were observed when unprotected sexual behavior was used as study outcomes.

Depending on its feasibility in other contexts, studies could use repeated sampling with PSA testing to facilitate objective measurement of sexual behavior over longer periods. Testing for Y-chromosome as an additional biomarker of recent sexual activity was not feasible in this study because its longer window of detection (i.e., up to 14 days) could have detected semen exposures that preceded study enrollment. ${ }^{24}$ However, given that the clearance and detection of PSA are unlikely to be strongly influenced by confounders, we believe that this biomarker provides an appropriate unbiased comparison of relative exposure to semen.

A strength of the trial was the high retention rate: $95 \%$ of randomized women were successfully observed and included in the intent-to-treat population. The high completion rate likely was a result of the short (6-day) interval required for follow-up combined with the use of active tracing of enrolled women. Women also might have been motivated to return to learn their STI test results. Another strength was the trial design, in which women were approached for consent for PSA testing of already collected vaginal swabs only after they had completed the quantitative interview at the follow-up visits. This temporality of procedures ensured that advance knowledge of biomarker testing did not alter participant behavior before their visit or influence their reporting of behaviors, although limited research suggests that advance knowledge that specimens will be tested for objective biomarkers might not influence participants' reports of recent unprotected sex. ${ }^{25}$

Finally, the study strongly benefited from the use of an objective biomarker for evaluating the effectiveness of the HIV/STI prevention counseling messages in addition to self-reported data. Beyond participants' actual sexual behavior, participant reporting of this behavior to investigators, for example, could have been influenced by the particular counseling message received. The trial also represents among the first efforts to incorporate testing of a semen biomarker in an HIV/STI intervention trial using a rapid, on-site assay, and its successful implementation demonstrates the feasibility of developing local capacity to conduct the inexpensive, on-site testing. ${ }^{11,26}$

In summary, we found no evidence supporting the superiority of promoting either abstinence or abstinence backed up by condoms in leading women to avoid unprotected intercourse during the treatment period for curable STIs and other reproductive tract infections. Treatment guidelines for STDs in the United States and Europe, for example, call for 
abstinence-only counseling in this situation to reduce risky sexual behavior. ${ }^{4,7}$ The evidence base underlying this recommendation for sexually active individuals is limited, however, and the current findings suggest that a comprehensive prevention message that includes condom use may also be appropriate.

\section{Acknowledgments}

The findings and conclusions in this report are those of the authors and do not necessarily represent the official position of the Centers for Disease Control and Prevention.

Supported by Women's Health and Fertility Branch, Division of Reproductive Health, Centers for Disease Control and Prevention, through a cooperative agreement maintained by US Agency for International Development with FHI 360 (Contraceptive and Reproductive Health Technology Research and Utilization Project, Agreement no.GPO-A-00-05-00022). Laboratory activities for the study were supported, in part, by the Southeastern Sexually Transmitted Infections Cooperative Research Center of the National Institute of Allergy and Infectious Diseases (U19-AI031496).

\section{REFERENCES}

1. World Health Organization. Global Prevalence and Incidence of Selected Curable Sexually Transmitted Infections. WHO; Geneva: 2001. Available at: http://www.who.int/hiv/pub/sti/ who_hiv_aids_2001.02.pdf. [Accessed November 17, 2012]

2. Sexton J, Garnett G, Røttingen J. Metaanalysis and metaregression in interpreting study variability in the impact of sexually transmitted diseases on susceptibility to HIV infection. Sex Transm Dis. 2005; 32:351-357. [PubMed: 15912081]

3. Røttingen J, Cameron DW, Garnett GP. A systematic review of the epidemiological interactions between classic sexually transmitted diseases and HIV: How much is really known? Sex Transm Dis. 2001; 28:579-597. [PubMed: 11689757]

4. Centers for Disease Control and Prevention. Sexually transmitted diseases treatment guidelines, 2010. MMWR Morb Mortal Wkly Rep. 2010; 59:RR-120.

5. Underhill K, Operario D, Montgomery P. Abstinence-only programs for HIV infection prevention in high-income countries. Cochrane Database Syst Rev. 2007; 4:CD005421. [PubMed: 17943855]

6. Underhill K, Montgomery P, Operario D. Abstinence-plus programs for HIV infection prevention in high-income countries. Cochrane Database Syst Rev. 2008; 1:CD007006. [PubMed: 18254124]

7. Radcliffe K. European STD guidelines. Int J STD AIDS. 2001; 12:S1-S102.

8. Braithwaite, AR. Practical Case Management of Common STI Syndromes. National HIV/STI Programme Ministry of Health; Jamaica: 2003. Revised version

9. Macaluso M, Lawson L, Akers R, et al. Prostate-specific antigen in vaginal fluid as a biologic marker of condom failure. Contraception. 1999; 59:195-201. [PubMed: 10382083]

10. Minnis AM, Steiner MJ, Gallo MF, et al. Biomarker validation of reports of recent sexual activity: Results of a randomized controlled study in Zimbabwe. Am J Epidemiol. 2009; 170:918-924. [PubMed: 19741042]

11. Hobbs MM, Steiner MJ, Rich KD, et al. Good performance of rapid prostate-specific antigen test for detection of semen exposure in women: Implications for qualitative research. Sex Transm Dis. 2009; 36:501-506. [PubMed: 19455082]

12. Huppert J, Mortensen JE, Reed JL, et al. Rapid antigen testing compares favorably to transcriptionmediated amplification assay for detection of Trichomonas vaginalis in young women. Clin Infect Dis. 2007; 45:194-198. [PubMed: 17578778]

13. Aho J, Koushik A, Diakité SL, et al. Biological validation of self-reported condom use among sex workers in Guinea. AIDS Behav. 2010; 14:1287-1293. [PubMed: 19680799]

14. Gallo MF, Behets FM, Steiner MJ, et al. Validity of self-reported 'safe sex' among female sex workers in Mombasa, Kenya-PSA analysis. Int J STD AIDS. 2007; 18:33-38. [PubMed: 17326860]

15. Gallo MF, Behets FM, Steiner MJ, et al. Prostate-specific antigen to ascertain reliability of selfreported coital exposure to semen. Sex Transm Dis. 2006; 33:476-479. [PubMed: 16865047] 
16. Pépin J, Fink GD, Khonde N, et al. Improving second-generation surveillance: The biological measure of unprotected intercourse using prostate-specific antigen in vaginal secretions of West African women. J Acquir Immune Defic Syndr. 2006; 42:490-493. [PubMed: 16773025]

17. Schulz KF, Altman DG, Moher D, et al. CONSORT 2010 statement: Updated guidelines for reporting parallel group randomized trials. Ann Intern Med. 2010; 152:726-732. [PubMed: 20335313]

18. Dreher M, Hudgins R. Maternal conjugal multiplicity and child development in rural Jamaica. Fam Relat. 2010; 59:495-505.

19. Lin, J.; Whitlock, E.; O'Connor, E., et al. Behavioral Counseling to Prevent Sexually Transmitted Infections. Agency for Healthcare Research and Quality; Rockville, MD: 2008.

20. Scott-Sheldon LA, Fielder RL, Carey MP. Sexual risk reduction interventions for patients attending sexually transmitted disease clinics in the United States: A meta-analytic review, 1986 to early 2009. Ann Behav Med. 2010; 40:191-204. [PubMed: 20652778]

21. Galvão LW, Oliveira LC, Díaz J, et al. Effectiveness of female and male condoms in preventing exposure to semen during vaginal intercourse: A randomized trial. Contraception. 2005; 71:130 136. [PubMed: 15707563]

22. Kamb ML, Fishbein M, Douglas JM Jr, et al. Efficacy of risk-reduction counseling to prevent human immunodeficiency virus and sexually transmitted diseases: A randomized controlled trial. Project RESPECT Study Group. JAMA. 1998; 280:116-117.

23. Figueroa JP, Duncan J, Byfield L, et al. A comprehensive response to the HIV/AIDS epidemic in Jamaica: A review of the past 20 years. West Indian Med J. 2008; 57:562-576. [PubMed: 19580238]

24. Brotman RM, Melendez JH, Smith TD, et al. Effect of menses on clearance of Y-chromosome in vaginal fluid: Implications for a biomarker of recent sexual activity. Sex Transm Dis. 2010; 37:14. [PubMed: 20118672]

25. Thomsen SC, Gallo MF, Ombidi W, et al. Randomized controlled trial on whether advance knowledge of prostate-specific antigen testing improves participant reporting of unprotected sex. Sex Transm Infect. 2007; 83:419-420. [PubMed: 17135328]

26. Mensch BS, Hewett PC, Abbott S, et al. Assessing the reporting of adherence and sexual activity in a simulated microbicide trial in South Africa: An interview mode experiment using a placebo gel. AIDS Behav. 2011; 15:407-421. [PubMed: 20886278] 


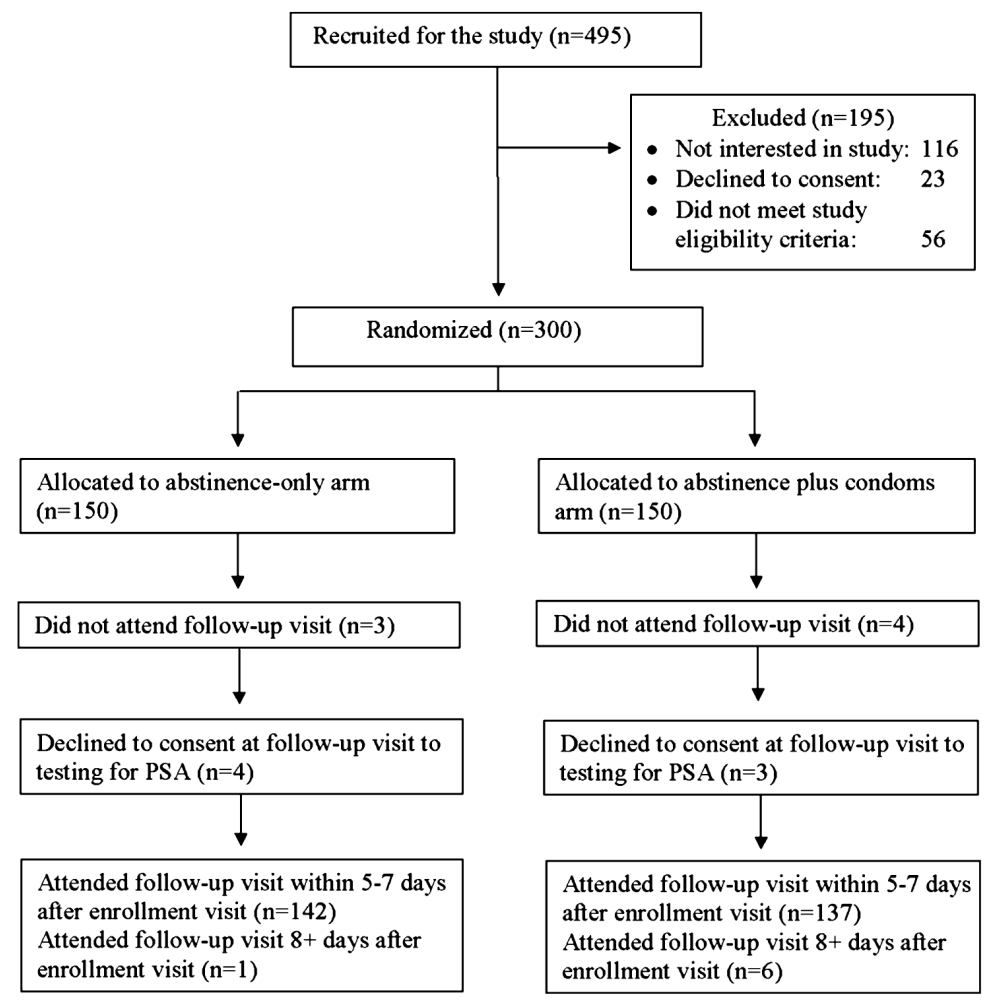

Figure 1.

Disposition of potential participants. 


\section{Panel}

Scripts for study counseling messages.

\begin{tabular}{|c|c|}
\hline \multirow[t]{3}{*}{ Abstinence } & $\begin{array}{l}\text { No sex. It is important that you do not have sex until after you have been checked by the doctor. You will see the } \\
\text { doctor again next week, when you return for your follow-up appointment. It is important that you take all of your } \\
\text { medication. It is important that you come back next week for your follow-up appointment. }\end{array}$ \\
\hline & $\begin{array}{l}\text { Sex may cause the medication to seem not to work because of reinfection or infection with other sexually } \\
\text { transmitted germs. }\end{array}$ \\
\hline & Sex may cause your partner(s) to be infected and make it possible for you to be infected again. \\
\hline \multirow[t]{5}{*}{ Abstinence plus condom } & $\begin{array}{l}\text { No sex. It is important that you do not have sex until after you have been checked by the doctor. You will see the } \\
\text { doctor again next week, when you return for your follow-up appointment. It is important that you take all of your } \\
\text { medication. It is important that you come back next week for your follow-up appointment. }\end{array}$ \\
\hline & If you are going to have sex, it is important that your partner uses a condom each time. \\
\hline & $\begin{array}{l}\text { Sex without a condom may cause the medication to seem not to work because of reinfection or infection with other } \\
\text { sexually transmitted germs. }\end{array}$ \\
\hline & Sex without a condom may cause your partner(s) to be infected and make it possible for you to be infected again. \\
\hline & Male condoms will be supplied and proper condom use explained. \\
\hline
\end{tabular}

Sex Transm Dis. Author manuscript; available in PMC 2014 February 01. 
TABLE 1

Characteristics and Behaviors at Enrollment by Counseling Arm for Intent-to-Treat Population $(\mathrm{N}=286)$

\begin{tabular}{|c|c|c|}
\hline Characteristic & Abstinence Only (n = 143) & Abstinence + Condom $(n=143)$ \\
\hline Age, median (IQR), y & $27(23-32)$ & $29(23-38)$ \\
\hline Parity, median (IQR) & $2(1-3)$ & $2(1-3)$ \\
\hline No. male partners in the past 3 mo, median (IQR), y & $1(1-2)$ & $1(1-2)$ \\
\hline \multicolumn{3}{|l|}{ Relationship status } \\
\hline Single & $42(29.4)$ & $39(27.3)$ \\
\hline Visiting partner ${ }^{*}$ & $52(36.4)$ & $54(37.8)$ \\
\hline Married or cohabiting & $49(34.3)$ & $50(35.0)$ \\
\hline \multicolumn{3}{|l|}{ Used a condom in the past $3 \mathrm{mo}$} \\
\hline Yes & $78(55.7)$ & $62(43.4)$ \\
\hline No & $62(44.3)$ & $81(56.6)$ \\
\hline \multicolumn{3}{|l|}{ Current contraceptive method } \\
\hline Condoms & $44(31.2)$ & $27(18.9)$ \\
\hline Injectable contraceptive & $26(18.2)$ & $28(19.6)$ \\
\hline Oral contraceptive & $13(9.1)$ & $15(10.5)$ \\
\hline Other & $19(13.3)$ & $20(14.0)$ \\
\hline None & $41(28.7)$ & $53(37.1)$ \\
\hline \multicolumn{3}{|l|}{ Self-reported vaginal sex in the past $2 \mathrm{~d}$} \\
\hline No vaginal sex & $111(77.6)$ & $106(74.1)$ \\
\hline Protected vaginal sex only & $12(8.4)$ & $11(7.7)$ \\
\hline$\geq 1$ unprotected vaginal act $>1$ laboratory-diagnosed STI at enrollment & $20(14.0)$ & $26(18.2)$ \\
\hline Yes & $54(38.0)$ & $56(39.7)$ \\
\hline No & $88(62.0)$ & $85(60.3)$ \\
\hline \multicolumn{3}{|l|}{ PSA detection } \\
\hline Yes & $10(7.0)$ & $14(9.9)$ \\
\hline No & $133(93.0)$ & $128(90.1)$ \\
\hline
\end{tabular}

Values are presented as no. (\%), unless otherwise indicated.

Understood as a conjugal partner who does not live with her. 
TABLE 2

Unprotected Sex Measured at Follow-up Visit by Counseling Arm for Intent-to-Treat Population $(\mathrm{N}=286)$

\begin{tabular}{|c|c|c|c|c|c|c|}
\hline & \multicolumn{2}{|c|}{ Abstinence + Condom } & \multicolumn{2}{|c|}{ Abstinence Only } & \multirow{2}{*}{$\begin{array}{l}\text { Risk Difference (95\% } \\
\text { CI) }\end{array}$} & \multirow{2}{*}{$\begin{array}{l}\text { Relative Risk (95\% } \\
\text { CI) }\end{array}$} \\
\hline & No. & $\%$ & No. & $\%$ & & \\
\hline \multicolumn{7}{|l|}{ Primary analysis } \\
\hline \multicolumn{7}{|l|}{ Positive for PSA } \\
\hline Yes & 17 & 11.9 & 12 & 8.4 & $3.5 \%(-3.5 \%-10.5 \%)$ & $1.42(0.70-2.86)$ \\
\hline No & 126 & 88.1 & 131 & 91.6 & & \\
\hline \multicolumn{7}{|l|}{ Secondary analyses } \\
\hline \multicolumn{7}{|l|}{ High positive for PSA } \\
\hline Yes & 11 & 7.7 & 8 & 5.6 & $2.1 \%(-3.7 \%, 7.9 \%)$ & $1.38(0.57-3.32)$ \\
\hline No & 132 & 92.3 & 135 & 94.4 & & \\
\hline \multicolumn{7}{|l|}{$\begin{array}{l}\text { Reported unprotected sex since } \\
\text { enrollment visit }\end{array}$} \\
\hline Yes & 25 & 17.9 & 16 & 11.7 & $6.2 \%(-2.1 \%, 14.5 \%)$ & $1.53(0.85-2.74)$ \\
\hline No & 115 & 82.1 & 121 & 88.3 & & \\
\hline \multicolumn{7}{|l|}{$\begin{array}{l}\text { Reported unprotected sex in } 2 \mathrm{~d} \\
\text { before follow-up visit }\end{array}$} \\
\hline Yes & 8 & 5.6 & 7 & 4.9 & $0.7 \%(-4.4 \%, 5.9 \%)$ & $1.15(0.43-3.09)$ \\
\hline No & 134 & 94.4 & 136 & 95.1 & & \\
\hline $\begin{array}{l}\text { No. of unprotected acts reported in } \\
2 \mathrm{~d} \text { before follow-up visit }\end{array}$ & \multicolumn{2}{|c|}{ Median, 0 ; range, $0-2$} & \multicolumn{2}{|c|}{ Median, 0 ; range, 0-3 } & - & - \\
\hline
\end{tabular}

